\title{
Successful Treatment of a Patient with Generalized Anxiety Disorder Using Dual-Site Transcranial Magnetic Stimulation
}

\author{
Richard C. Holbert ${ }^{1,2}$, Daniel P. Witter ${ }^{3 *}$ \\ ${ }^{1}$ Assistant Professor, University of Florida College of Medicine, Department of Psychiatry, USA \\ ${ }^{2}$ Medical Director of University of Florida Psychiatric Hospital, USA \\ ${ }^{3}$ PGY4, University of Florida College of Medicine, Department of Psychiatry, USA
}

*Corresponding author: Daniel P.Witter, M.D., Ph.D, 4037 NW 86th Terrace, UF Health Adult, Psychiatry, Gainesville, FL 32606, USA, Email: dwitter@ufl.edu

\begin{abstract}
The use of Transcranial Magnetic Stimulation (TMS) in treatment-resistant cases of certain psychiatric illnesses is an ever-expanding field of research. As our understanding of the neurobiological underpinnings of psychiatric illness increases, abnormal function in neurocircuits guides the choice of unique targets for neurostimulation therapy. Generalized Anxiety Disorder (GAD) is a common psychiatric disorder with high rates of treatment-resistance and putative functional abnormalities in a variety of frontal and limbic circuits. This case highlights the results of a novel, dual-site TMS protocol in a 25 -year-old female with treatment-resistant GAD. The seven week course of treatment combined low frequency TMS over the dorsolateral right prefrontal cortex and high frequency TMS over the dorsolateral left prefrontal cortex. This method of treatment resulted in a 91\% improvement in GAD symptoms based on by the Hamilton Anxiety Rating Scale and considerable overall functional improvement based on the Clinical Global Impression-Severity scale. We propose that TMS may prove useful for treatment-resistant GAD and that targeting multiple sites with TMS may improve outcomes compared to TMS targeting a single site.
\end{abstract}

Received Date: September 28, 2016

Accepted Date: November 14, 2016

Published Date: November 21, 2016

Citation: Witter, D.P., et al. Successful Treatment of a Patient with Generalized Anxiety Disorder using Dual-Site Transcranial Magnetic Stimulation. (2016) Int J Neuro Brain Dis 3(4): 1- 3.

DOI: $10.15436 / 2377-1348.16 .1143$

\section{Introduction}

Generalized Anxiety Disorder (GAD) is a chronic mental illness that has pervasive negative effects on social and occupational functioning and quality of life. It is characterized by persistent and excessive worry accompanied by distressing physical symptoms including restlessness, fatigue, difficulty concentrating, irritability, muscle tension, and sleep disturbances. The lifetime prevalence is around $5 \%$, with women affected twice as commonly as men ${ }^{[1,2]}$. Treatments include a variety of psychopharmacologic agents and psychotherapy. Selective serotonin reuptake inhibitors, serotonin norepinephrine reuptake inhibitors, tricyclic antidepressants, and benzodiazepines have demonstrated benefit, as has cognitive behavioral therapy. The symptoms of GAD do not always respond to these treatments, however, and nearly half of patients have inadequate symptom resolution or complete lack of response $\mathrm{e}^{[3-5]}$. Additional effective treatments for GAD would be a boon to mental health practitioners and their patients.
Transcranial Magnetic Stimulation (TMS) is the utilization of a time-varying magnetic field to non-invasively induce an electric current to stimulate or inhibit neuronal activity. The Food and Drug Administration has approved TMS for treatment resistant Major Depressive Disorder (MDD). It has further been used experimentally to treat other psychiatric disorders, including panic disorder, obsessive compulsive disorder, social phobia, and smoking cessation ${ }^{[6-8]}$. While the exact mechanism of TMS remains unknown, it is thought to have a therapeutic modulating effect on neuro-circuits involved in psychiatric disorders ${ }^{[9]}$. High frequency stimulation (generally accepted as $>5 \mathrm{~Hz}$ ) is considered to be excitatory, and low frequency stimulation $(\leq 1 \mathrm{~Hz})$ is considered to be inhibitory ${ }^{[10,11]}$. Total pulse number may correlate to symptom improvement, although this has not yet been determined.

As with many psychiatric disorders, anxiety is thought to be due to functional abnormalities within the cortico-stria- 
tal-cortical neurocircuitry ${ }^{[4]}$. Neuroimaging studies have indicated abnormalities in the prefrontal cortex region of patients with anxiety ${ }^{[12,13]}$. Furthermore, hyperactivity of the right Dorsolateral Prefrontal Cortex (DLPFC) has been implicated in anxious symptoms, and hypoactivity of the left DLPFC has been associated with abnormal emotional and memory processing in anxious patients ${ }^{[14,15]}$. A few exploratory studies have reported improvement in post-traumatic stress disorder and panic disorder symptoms using TMS targeting the right DLPFC ${ }^{[8,16]}$. Given the encouraging results of these studies, GAD is now also being examined as an anxiety disorder that may respond to neurostimulation. One open-label case series indicated improvement in GAD symptoms using low frequency TMS over the right prefrontal cortex ${ }^{[4]}$. Another study reported symptom improvement in patients with comorbid GAD and MDD treated with TMS targeting the right DLPFC in addition to the left DLPFC, the latter being the most commonly used site for TMS in MDD ${ }^{[17]}$. In this case report, we describe the successful treatment of GAD symptoms leading to significant improvement in social functioning using low frequency (inhibitory) stimulation of the right DFPLC and high frequency (excitatory) stimulation at the left DFPLC. Of note, we also used a higher total pulse number than is generally reported for TMS studies.

\section{Case Report}

Our patient was a 25 -year-old female who presented with symptoms of anxiety and generalized worry since early adolescence accompanied by severe muscle tension, decreased concentration, and easy fatigue. She was living alone at the time of the study with financial support from her parents and was essentially housebound due to her anxiety. She met criteria for GAD at the time of the study based on a diagnostic interview as per the DSM- $5^{[18]}$. Her initial Hamilton Anxiety Rating Scale (HAM-A) ${ }^{[19]}$ score was 32 . While she denied depression at the time of the study, she had been diagnosed with MDD in the past. She scored a nine on her initial Montgomery Asberg Depression Rating Scale (MADRS) ${ }^{[20]}$ and a five on the Clinical Global
Impression-Severity scale (CGI-S) ${ }^{[21]}$. She had no other comorbidpsychiatric or substance use disorders or medical issues. She was taking extended-release venlafaxine 225 milligrams daily prior to the study and continued this medication during TMS treatment.

TMS was performed using the Neuronetics Neuro Star(NNS) TMS System after appropriate informed consent was obtained. Motor Threshold (MT) was determined using the NNS Therapy System standardized method. The patient received a total of 40 daily treatments. For the first four weeks, she received 19 treatments of 1200 pulses of low frequency TMS at $1 \mathrm{~Hz}$ and $120 \%$ MT over the right DLPFC. The rating scales above were used to assess symptoms throughout the course of treatment. Scores are presented in Table 1. The patient began to show some benefit at the end of the second week of treatment. She reported feeling less anxious and tense and was more interactive with her family and friends. These initial improvements appeared to plateau by the fourth week of treatment. It was decided at this time to also treat the left DLPFC with high frequency TMS. While this generally means a frequency of $\geq 5 \mathrm{~Hz}$, she was only able to tolerate $3 \mathrm{~Hz}$ due to discomfort. The discomfort from the $3 \mathrm{~Hz}$ quickly resolved but she did not want to attempt to increase it. The remaining 21 treatments were performed with 1200 pulses at $1 \mathrm{~Hz}$ and $120 \%$ MT over the right dorsolateral lateral prefrontal cortex and 1800 pulses at three and $120 \%$ MT over the left dorsolateral prefrontal cortex. The patient showed continued improvement during her treatment until the end of the eightweek course of treatment. In total, 48,000 pulses at $1 \mathrm{~Hz}$ were delivered to the right dorsolateral prefrontal cortex and 37,800 pulses at $3 \mathrm{~Hz}$ were delivered to the left dorsolateral prefrontal cortex. HAM-A scores decreased $91 \%$ over the course of treatment. MADRS scores indicated an improvement of $89 \%$. CGI-S score showed a $60 \%$ improvement. Her day-to-day functioning had markedly improved by the end of the treatment, and she was able to fully engage with family and friends and had even begun to look for a job. She was ultimately lost to subsequent follow up as she lived in a different city to which she returned following the study.

Table 1: Scores of Ratings Scales throughout Treatment.

\begin{tabular}{|l|c|c|c|c|c|c|c|c|c|}
\hline & $\begin{array}{c}\text { Prior to } \\
\text { Treatment }\end{array}$ & Week 1 & Week 2 & Week 3 & Week 4 & Week 5 & Week 6 & Week 7 & $\begin{array}{c}\text { End of } \\
\text { Treatment }\end{array}$ \\
\hline HAM-A & 32 & 30 & 23 & 12 & 12 & 10 & 9 & 4 & 3 \\
\hline MADRS & 9 & & & & & & & 1 \\
\hline CGI-S & 5 & & & & & & & & \\
\hline
\end{tabular}

HAM-A: Hamilton Anxiety Rating Scale. MADRS: Montgomery Asberg Depression Rating Scale. CGI-S Clinical Global Impression-Severity scale

As our understanding of psychiatric disorders evolves, abnormalities in neuro circuits continue to be implicated. TMS has emerged as a non-invasive tool to modulate a faulty circuit by targeting a specific brain region within the circuit. Individual circuits do not act independently of other circuits, however, and there is frequently overlap between circuit components or modulation of one circuit by another. Based upon the theoretical neurobiological underpinnings of anxiety as well as the results of the few, but growing number of studies of TMS in GAD, we targeted both the right and left DLPFCs in this case. Furthermore, acting on the hypothesis that pulse number and symptom im- provement may be correlated, we also provided a greater number of total pulses than are typically reported in TMS cases. This unique protocol of dual-site TMS utilizing a greater total pulse number produced significant symptomatic relief in our patient with GAD. While larger trials need to be completed to further substantiate these results, we suggest that TMS may be a potentially beneficial clinical therapy for treatment-resistant GAD and that dual-site TMS may provide improved results compared to the standard single-site model of TMS in psychiatric disorders. Finally, additional studies would be beneficial in elucidating the relationship between pulse number and symptom improvement. 


\section{References}

1. Kessler, R.C., Petukhova, M., Sampson, N.A., et al. Twelve-month and lifetime prevalence and lifetime morbid risk of anxiety and mood disorders in the United States. (2012) Int J Methods Psychiatr Res. 21(3): 169-184.

2. Weisberg, R.B. Overview of generalized anxiety disorder: epidemiology, presentation, and course. (2009) J Clin Psychiatry 70 Suppl 2: 4-9. 3. Bandelow, B., Boerner, J.R., Kasper, S., et al. The diagnosis and treatment of generalized anxiety disorder. (2013) Dtsch Arztebl Int. 110(17): 300-309.

4. Bystritsky, A., Kaplan, J.T., Feusner, J.D., et al. A preliminary study of fMRI-guided rTMS in the treatment of generalized anxiety disorder. (2008) J Clin Psychiatry 69(7): 1092-1098.

5. Yonkers, K.A., Warshaw, M.G., Massion, A.O., et al. Phenomenology and course of generalised anxiety disorder. (1996) Br J Psychiatry 168(3): 308-313.

6. Berlim, M.T., Neufeld, N.H., Van, den, Eynde, F. Repetitive transcranial magnetic stimulation (rTMS) for obsessive-compulsive disorder (OCD): an exploratory meta-analysis of randomized and sham-controlled trials. (2013) J Psychiatr Res 47(8): 999-1006.

7. Yang, L.Z., Yang, Z., Zhang, X. Non-invasive Brain Stimulation for the Treatment of Nicotine Addiction: Potential and Challenges. (2016) Neurosci Bull.

8. Zwanzger, P., Fallgatter, A.J., Zavorotnyy, M., et al. Anxiolytic effects of transcranial magnetic stimulation--an alternative treatment option in anxiety disorders? (2009) J Neural Transm (Vienna) 116(6): 767-775.

9. Lewis, P.M., Thomson, R.H., Rosenfeld, J.V., et al. Brain Neuromodulation Techniques: A Review. (2016) Neuroscientist 22(4): 406-421.

10. Baudewig, J., Siebner, H.R., Bestmann, S., et al. Functional MRI of cortical activations induced by transcranial magnetic stimulation (TMS). (2001) Neuroreport 12(16): 3543-3548.
11. Bestmann, S., Baudewig, J., Siebner, H.R., et al. Subthreshold high-frequency TMS of human primary motor cortex modulates interconnected frontal motor areas as detected by interleaved fMRI-TMS. (2003) Neuroimage 20(3): 1685-1696.

12. Moon, C.M., Kim, G.W., Jeong, G.W. Whole-brain gray matter volume abnormalities in patients with generalized anxiety disorder: voxel-based morphometry. (2014) Neuroreport 25(3): 184-189.

13. Schienle, A., Ebner, F., Schafer, A. Localized gray matter volume abnormalities in generalized anxiety disorder. (2011) Eur Arch Psychiatry Clin Neurosci 261(4): 303-307.

14. Balconi M, Ferrari C. rTMS stimulation on left DLPFC affects emotional cue retrieval as a function of anxiety level and gender. (2012) Depress Anxiety 29(11): 976-982.

15. Balconi M, Ferrari C. Left DLPFC rTMS stimulation reduced the anxiety bias effect or how to restore the positive memory processing in high-anxiety subjects. (2013) Psychiatry Res 209(3): 554-559.

16. Van, Honk, J., Tuiten, A., van, den, Hout, M., et al. Baseline salivary cortisol levels and preconscious selective attention for threat. A pilot study. (1998) Psychoneuroendocrinology 23(7): 741-747.

17. White, D., Tavakoli, S. Repetitive transcranial magnetic stimulation for treatment of major depressive disorder with comorbid generalized anxiety disorder. (2015) Ann Clin Psychiatry 27(3): 192-196.

18. American Psychiatric Association. Diagnostic and statistical manual of mental disorders. ed. Washington, DC2013.

19. Hamilton M. The assessment of anxiety states by rating. (1959) $\mathrm{Br}$ J Med Psychol 32(1): 50-55.

20. Montgomery, S.A., Asberg, M. A new depression scale designed to be sensitive to change. (1979) Br J Psychiatry 134: 382-389.

21. Guy, W. Patient assessment in clinical trials. (1982) Prog Neuropsychopharmacol Biol Psychiatry 6(4-6): 601-606.
Online ISSN: $2377-1348$

Journal Title: International Journal Neurology and Brain Disorders Journal Short Name: Int J Neurol Brain Disord
Ommega Online Publishers

E-mail: neurology@ommegaonline.com

Website: www.ommegaonline.org 